\title{
Characteristics of fecal gut microbiota in patients with colorectal cancer at different stages and different sites
}

\author{
QINSONG SHENG ${ }^{1}$, HAIXIA DU $^{2}$, XIAOFEI CHENG ${ }^{1}$, XIAOBIN CHENG $^{1}$, YUNNING TANG $^{3}$, \\ LELIN PAN $^{1}$, QUNMIN WANG ${ }^{1}$ and JIANJIANG LIN ${ }^{1}$ \\ ${ }^{1}$ Department of Colorectal and Anal Surgery, The First Affiliated Hospital of College of Medicine, \\ Zhejiang University, Hangzhou, Zhejiang 310003; ${ }^{2}$ Institute of Cardio-Cerebrovascular Diseases, \\ Zhejiang Chinese Medical University, Hangzhou, Zhejiang 310053; ${ }^{3}$ Department of Colorectal and Anal Surgery, \\ Changshan People' Hospital, Changshan, Zhejiang 324200, P.R. China
}

Received November 27, 2018; Accepted August 1, 2019

DOI: $10.3892 / 01.2019 .10841$

\begin{abstract}
Numerous studies have revealed that the gut microbiota serves an important role in the pathogenesis of colorectal cancer (CRC). The present study aimed to investigate the populations present in the gut microbiota in patients with CRC of different stages and at different sites. Fecal samples were obtained from $67 \mathrm{CRC}$ patients and 30 healthy controls, which were analyzed by sequencing the V3-V4 region of the $16 \mathrm{~S}$ rRNA gene. Increased diversity of the fecal gut microbiota in patients with CRC was reported compared with the healthy controls. In the present study, at the genus level, the relative abundances of Prevotella, Collinsella and Peptostreptococcus in the gut microbiota of CRC patients were substantially increased compared with healthy controls, while the relative abundance of Escherichia-Shigella was significantly lower. In addition, differences in the fecal gut microbiota were also compared between patients with stage I-IV CRC and healthy controls. The results revealed that the abundances of the genera Peptostreptococcus, Collinsella and Ruminococcus were significantly increased in patients with CRC stage I compared with the healthy controls, while Alistipes was enriched in patients with stage III CRC compared with patients with stage IV. Furthermore, the present study reported that the genera Veillonella and Coprobacter were more abundant in the proximal segments than in the distal segments of the colon. In conclusion, despite the low number of samples employed in the present study, a signature of genera indicating dysbiosis of the gut microbiota of patients with stage I-IV CRC patients was proposed, which may provide insight into the mechanisms
\end{abstract}

Correspondence to: Dr Jianjiang Lin, Department of Colorectal and Anal Surgery, The First Affiliated Hospital of College of Medicine, Zhejiang University, 79 Qingchun Road, Hangzhou, Zhejiang 310003, P.R. China

E-mail: linjianjiang@zju.edu.cn

Key words: colorectal cancer, gut microbiota, 16S rRNA sequencing, bioinformatics analysis underlying the progression of CRC. These findings are also valuable for developing novel fecal diagnostic methods and therapeutic strategies for the treatment of CRC.

\section{Introduction}

Colorectal cancer (CRC) is a common life threatening disease worldwide and is also one of the most common causes of cancer-associated mortality in developed countries $(1,2)$. In addition, 700,000 people succumb to this disease annually, making it the fourth most deadly cancer among males and females (3). The development of CRC is a heterogeneous process involving numerous genetic and epigenetic variations, which are affected by environmental conditions, diet, inflammatory responses and microbial adhesion; however, the etiology of CRC remains unknown (4). Previously, gut microbial dysbiosis has been reported to serve a vital role in homeostasis and the development of CRC and has received increasing attention in this research field $(5,6)$. Therefore, improved understanding of the differential combination of microorganisms that comprise the gut microbiota may provide novel insight for developments in the treatment of CRC.

Further investigation into the interactions between host microbes is required to understand how the gut microbiota promotes disease progression. Based on differences in embryology, morphology and anatomy, Bufill (7) proposed the existence of distinct types of CRC according to the location of the tumor relative to the proximal (right) or distal (left) segments of the splenic flexor muscle in the colon. Epidemiological studies revealed differences between these proximal and distal segments $(8,9)$. The association between the gut microbiota and the initiation and progression of CRC is well reported (10). Alterations in gut microecology, which is linked to disease progression, may be applied for the diagnosis of gastrointestinal diseases. Of note, Escherichia-Shigella has been reported to be linked to the development of CRC and variations in conditional pathogens, which may be the main cause of gut microbial dysbiosis in patients with CRC $(11,12)$. Prevotella has been associated with the expression of Th17 response-related genes and correlated with the reduced survival of CRC patients (13). Additionally, studies into the 
fecal microbiota may improve understanding of the general composition of the gut microbiota and its imbalance under different conditions $(14,15)$.

Understanding the factors that affect the composition of the microbial community in the gut is essential for developing $\mathrm{CRC}$ treatments. In the present study, the V3-V4 region of $16 \mathrm{~S}$ rRNA was sequenced using an Illumina sequencing platform to analyze the composition of the gut microbiota of fecal samples from 67 patients with CRC and 30 healthy controls. The present study also aimed to investigate differences in the gut microbiota among various stages of CRC and between distal and proximal segments of the colon in CRC.

\section{Materials and methods}

Patients and samples. In total, 67 patients with CRC (aged 33-86 years, 42 males and 25 females) and 30 healthy controls (aged 27-79 years, 15 males and 15 females) were recruited between October 2016 and August 2017 from The First Affiliated Hospital of Zhejiang University (Hangzhou, China). All patients with CRC were classified according to their postoperative clinical data, using the tumor-node-metastasis (TNM) staging system for malignant tumors (16). The CRC patients were selected according to the following criteria: No complications (such as chronic bowel disease, diabetes, other signs of infections or hypertension); no family history of CRC or recurrence of CRC, no radiotherapy and chemotherapy prior to surgical resection; no use of antibiotics, non-steroidal anti-inflammatory drugs, statins or probiotics within the past 3 months prior to the collection of stool samples; no food allergies and no potential immunodeficiency. The 30 healthy individuals were selected as controls and were matched according to sex and age during a routine physical examination; healthy controls corresponded to stage $\mathrm{O}$ of the staging system applied for classifying CRC patients. In addition, the healthy controls did not have gastrointestinal tract disorders or other complications and were not administered antibiotics or probiotics during the 3 months prior to sample collection. The present study was approved by the Research Ethics Committee of The First Affiliated Hospital, College of Medicine, Zhejiang University (approval no. 2016-436). All patients indicated that they had obtained written informed consent in the present study. On the morning of the day prior to surgical resection, one fecal sample was collected in a sterile container from each healthy control and patient with CRC. The data recorded included the general information (age and sex) and the clinical data of patients (tumor stages, tumor sites and pathological data). Fecal samples were frozen immediately after collection and stored at $-80^{\circ} \mathrm{C}$ until DNA was extracted.

DNA extraction. Genomic DNA was extracted from each fecal sample ( $200 \mathrm{mg}$ ) using the QIANamp DNA Stool Mini kit (Tiangen Biotechnology Co., Ltd.) according to the manufacturer's protocols $(15,17)$. The concentration and quality of the extracted DNA were determined using a NanoDrop spectrophotometer; the integrity of the DNA samples was analyzed via $2 \%(\mathrm{w} / \mathrm{v})$ agarose gel electrophoresis. All DNA samples were stored at $-80^{\circ} \mathrm{C}$ until use for microbial characterization.

Polymerase chain reaction (PCR) amplification and sequencing. The extracted DNA samples were sent to an external company (Jingbai Biotechnology Co., Ltd.) for library construction and sequencing using the paired-end protocol on the MiSeq Illumina platform. The V3-V4 region of $16 \mathrm{~S}$ rRNA was amplified by PCR. The sequences for the universal primers (V3-V4) were: Forward, (515F) 5'-TCGTCGGCA GCGTCAGATGTGTATAAGAGACAGCCTACGGGNGGC WGCAG-3' and reverse, (806R) 5'-GTCTCGTGGGCTCGG AGATGTGTATAAGAGACAGGACTACHVGGGTATCTA

ATCC-3'. Briefly, PCR was performed using a reaction volume of $50 \mu \mathrm{l}$ containing $10 \mathrm{ng}$ DNA template, $25 \mu \mathrm{l}$ 2X Phanta Max Master Mix buffer solution (Vazyme Biotech Co., Ltd), $2 \mu 1$ each primer $\left(10 \mathrm{M}\right.$ ) and $\mathrm{ddH}_{2} \mathrm{O}$ (to $50 \mu \mathrm{l}$ ). Amplifications were performed under the following conditions: Initial denaturation at $95^{\circ} \mathrm{C}$ for $3 \mathrm{~min}$, followed by 25 cycles of denaturation at $95^{\circ} \mathrm{C}$ for $30 \mathrm{sec}$, primer annealing at $55^{\circ} \mathrm{C}$ for $30 \mathrm{sec}$ and extension at $72^{\circ} \mathrm{C}$ for $45 \mathrm{sec}$, followed by final elongation at $72^{\circ} \mathrm{C}$ for $5 \mathrm{~min}$. The PCR products were purified using the MiniE-lute PCR purification kit (Axygen; Thermo Fisher Scientific, Inc.) and quantified using a detection system (Light Cycler ${ }^{\circledR} 96$ Flex Real-time PCR System; Roche Diagnostics). Then, samples were pooled at equal concentrations. The thermocycling conditions were as follows: $95^{\circ} \mathrm{C}$ for $3 \mathrm{~min}$, followed by 8 cycles of denaturation at $95^{\circ} \mathrm{C}$ for $30 \mathrm{sec}$; primer annealing at $55^{\circ} \mathrm{C}$ for $30 \mathrm{sec}$ and extension at $72^{\circ} \mathrm{C}$ for $45 \mathrm{sec}$ and final elongation at $72^{\circ} \mathrm{C}$ for $5 \mathrm{~min}$. Library construction and sequencing with specific tags were performed on a MiSeq Benchtop Sequencer (Illumina, Inc.).

Bioinformatics analyses. Based on the overlap of paired-end reads, the microbial data obtained via the Illumina platform were optimized and the paired reads were combined into sequences. The quality of reads and the merged results were filtered as follows: The maximum mismatch ratio of the overlap portion was 0.15 ; the minimum overlap of merging paired reads was $10 \mathrm{bp}$ and an average of $<20$ bases at the end of reads and sequences other than 300-480 bp in length were filtered. In order to obtain high-quality and more accurate biological information, sequences containing some point mutations and macromolecular homopolymers were used (Qiime, version 1.17 http://qiime.org/) (18). Then, the selected sequences were clustered into optional taxonomic units (OTUs) using Usearch (version 7.1, http://drive5. com/uparse/) with a standard similarity of $\geq 97 \%$. The chimera sequences generated by PCR amplification were detected and excluded using Uchime (version 4.2.40 http://drive5.com/usearch/manual/uchime_algo.html) (19). The representative OTUs were compared with the optimized sequences to obtain the abundance of OTUs within each sample for subsequent analysis.

Richness estimators (Ace, Chao) and $\alpha$-diversity estimators (Shannon and Simpson indexes) were calculated using the mothur software package (version 1.35.1) (15). Unweighted Unifrac distance metrics analysis of relative abundance at different levels in each sample was conducted using OTUs and principal coordinate analysis (PCOA) was performed to demonstrate the clustering of different samples according to the distance matrix (20). This method takes into account the divergence between different sequences. In addition, $\mathrm{R}$ package (version 2.15.3; R Foundation for Statistical Computing) was also employed for other statistical analyses. 
Table I. Clinicopathological characteristics of patients with CRC and healthy controls enrolled in the present study.

\begin{tabular}{lccc}
\hline Characteristic & Male & Female & Total \\
\hline CRC, $\mathrm{n}$ & 42 & 25 & 67 \\
Age, years & $63.69 \pm 12.85$ & $61.32 \pm 10.37$ & $62.81 \pm 11.96$ \\
Tumor stage & & & \\
I & 12 & 6 & 18 \\
II & 7 & 10 & 17 \\
III & 19 & 8 & 27 \\
IV & 4 & 1 & 5 \\
Tumor site & & & \\
Distal (left) & 32 & 12 & 44 \\
segment & & & \\
Proximal (right) & 10 & 13 & 23 \\
segment & & & \\
Healthy controls, $\mathrm{n}$ & 15 & 15 & 30 \\
Age, years & $51.79 \pm 14.14$ & $50.14 \pm 16.28$ & $50.96 \pm 14.99$
\end{tabular}

CRC, colorectal cancer.

Based on the Kyoto Encyclopedia of Genes and Genomes (KEGG) database, the functional classifications in the 16 sRNA genes of fecal gut microbiota were predicted by the software Phylogenetic Investigation of Communities by Reconstruction of Unobserved States (PICRUSt). PICRUSt was used online in the Galaxy workflow framework.

Statistical analyses. As the majority of the datasets did not meet the assumptions of normal distribution, non-parametric Dunn's tests with Kruskal-Wallis tests or Mann-Whitney U test where applicable were used, and analysis of variance were performed to analyze differences in relative expression between patients with CRC and healthy controls using GraphPad Prism 6.00 (GraphPad Software, Inc.). Unless otherwise indicated, data were expressed as the mean \pm standard deviation. $\mathrm{P}<0.05$ was considered to indicate a statistically significant difference.

\section{Results}

Clinicopathological status of patients. A total of 67 patients with CRC were enrolled in the present study, including 42 males and 25 females. The diagnosis of CRC was assessed by two experienced pathologists. The average age of males was $63.69 \pm 12.85$ years and that of females was $61.32 \pm 10.37$ years. All patients with CRC were divided into stages I-IV:18 as stage I, 17 as stage II, 27 as stage III and 5 as stage IV. The 30 healthy controls were considered as stage $\mathrm{O}$, in accordance with the staging system employed. In addition, the number of proximal (right) and the distal (left) segments collected were 23 and 44, respectively. The clinical and pathological characteristics of patients with CRC are presented in Table I.

Richness and diversity analysis. In total, 32,992 and 32,704 high-quality and usable reads were obtained from fecal samples of 67 patients with CRC and 30 healthy controls from sequencing the 16S rRNA gene, with an average length of 416 and $418 \mathrm{bp}$, respectively. At the level of $3 \%$ difference, there were 531,164 OTUs in all samples, with an average of 149,755 OTUs ( $\mathrm{n}=97)$ per sample. The Good's coverage value of each group was $>93 \%$, indicating that the $16 \mathrm{~S}$ rRNA sequences identified in each group represented the majority of bacteria present in the samples analyzed. The Chaol index was used to evaluate microbial richness; the Shannon and Simpson diversity indexes were applied to assess the diversity of fecal gut microbiota between patients with CRC and healthy controls. The results revealed that the Shannon and Simpson diversity indexes (Shannon, $4.63 \pm 0.92$ vs. $4.12 \pm 1.09 ; \mathrm{P}=0.015$; Simpson, $0.86 \pm 0.11$ vs. $0.79 \pm 0.15 ; \mathrm{P}=0.011)$ of $\mathrm{CRC}$ patients were significantly increased compared with the healthy controls. This indicated that the microbiota diversity of patients with CRC patients was significantly increased compared with the healthy controls; no significant difference in the Chaol index $(1,659 \pm 600$ vs. $1,763 \pm 722 ; P=0.45)$ between $C R C$ patients and healthy controls was observed. Furthermore, there were no significant differences in the Chaol, Shannon diversity and Simpson diversity indexes among the gut microbiota of patients with CRC stages I-IV and healthy controls ( $\mathrm{P}>0.05$; Fig. 1).

Bacteria comprising the fecal microbial community of healthy controls and patients with CRC. The relative abundance of dominant bacterial phyla for each fecal sample was presented in Fig. S1. At the phylum level, dominant bacterial phyla were analyzed and presented according to relative abundance. The present study reported that all bacterial phyla were detected from the interpretable sequences among patients with CRC and the healthy controls. The first five dominant bacterial phyla were Firmicutes, Bacteroidetes, Proteobacteria, Verrucomicrobia and Actinobacteria in the CRC patients and healthy controls. Among these top 20 dominant bacterial phyla, no significant differences in the fecal gut microbiota were observed within patients with CRC of stages I-IV and healthy controls (Fig. S1).

The relative abundance of the dominant bacterial genera within each fecal sample was presented in Fig. 2. At the genus level, the dominant bacterial genera were analyzed and presented according to relative abundance. The results revealed that all bacterial genera were detected from the interpretable sequences among patients with CRC and healthy controls; the first five dominant bacterial were Escherichia-Shigella, Bacteroides, Faecalibacterium, Prevotella and Akkermansia in the CRC patients and healthy controls. Among these dominant bacterial genera, according to the analysis of the top 15 genera, the abundance of Prevotella differed within the fecal gut microbiota of patients with CRC of stages I-IV and healthy controls (Fig. 2).

Differences in fecal microbial communities between healthy controls and CRC patients based on classification comparisons. Principal component analysis (PCA) and PCoA were performed according to the relative abundance of OTUs in each group, and the composition of the gut microbiota of all samples was compared. Distance-based PCA revealed the relative abundances of the fecal microbial community among patients with CRC of stages I-IV and healthy controls, which were separated by the first two principal component scores 

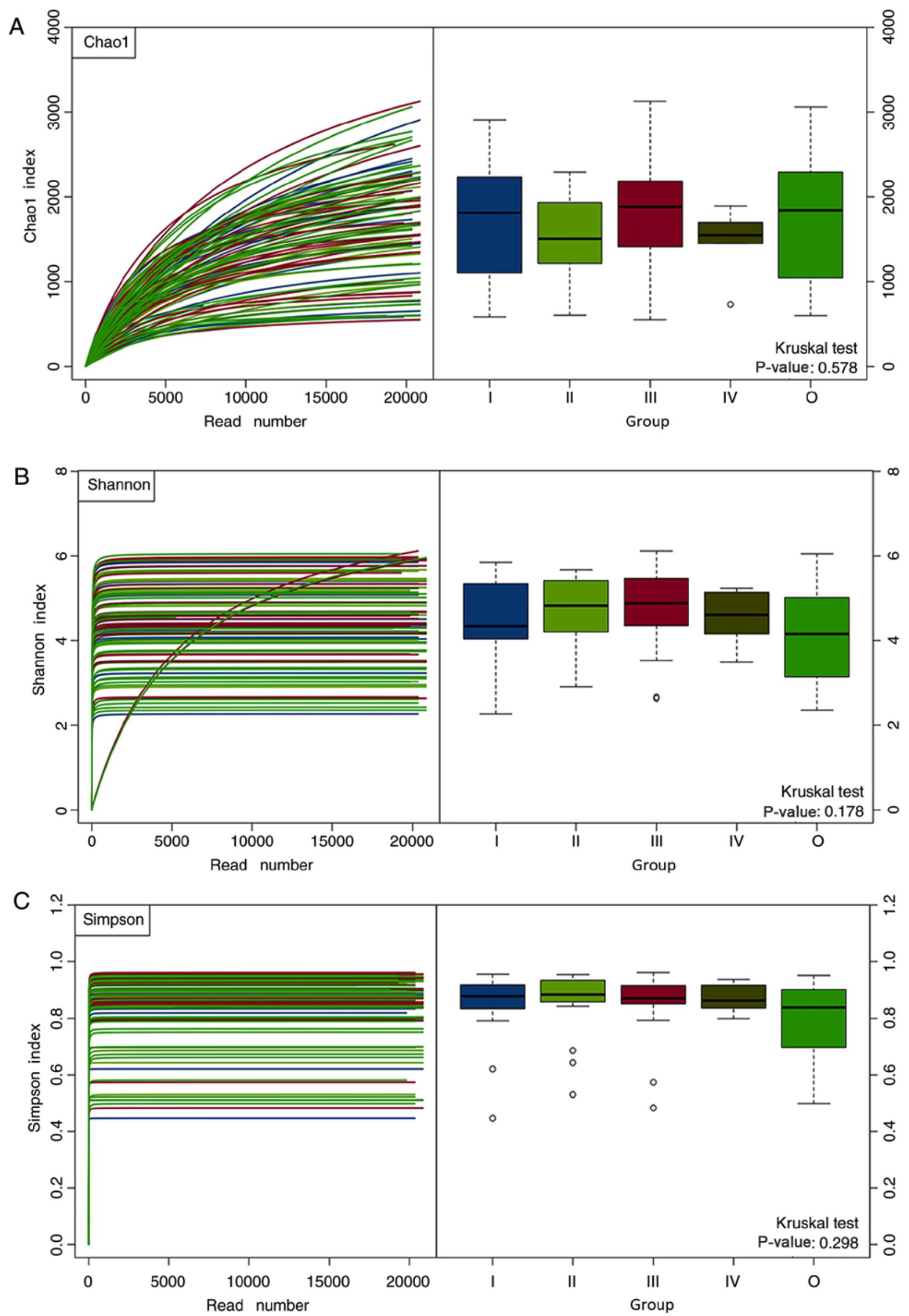

Figure 1. Richness and diversity analysis of 16S rRNA gene sequences obtained from fecal samples. Lines represent the mean and error bars indicate the standard deviation. Comparisons between the (A) Chaol, (B) Shannon diversity and (C) Simpson diversity indexes of patients with colorectal cancer of stages I, II, III and IV and healthy controls. 
A
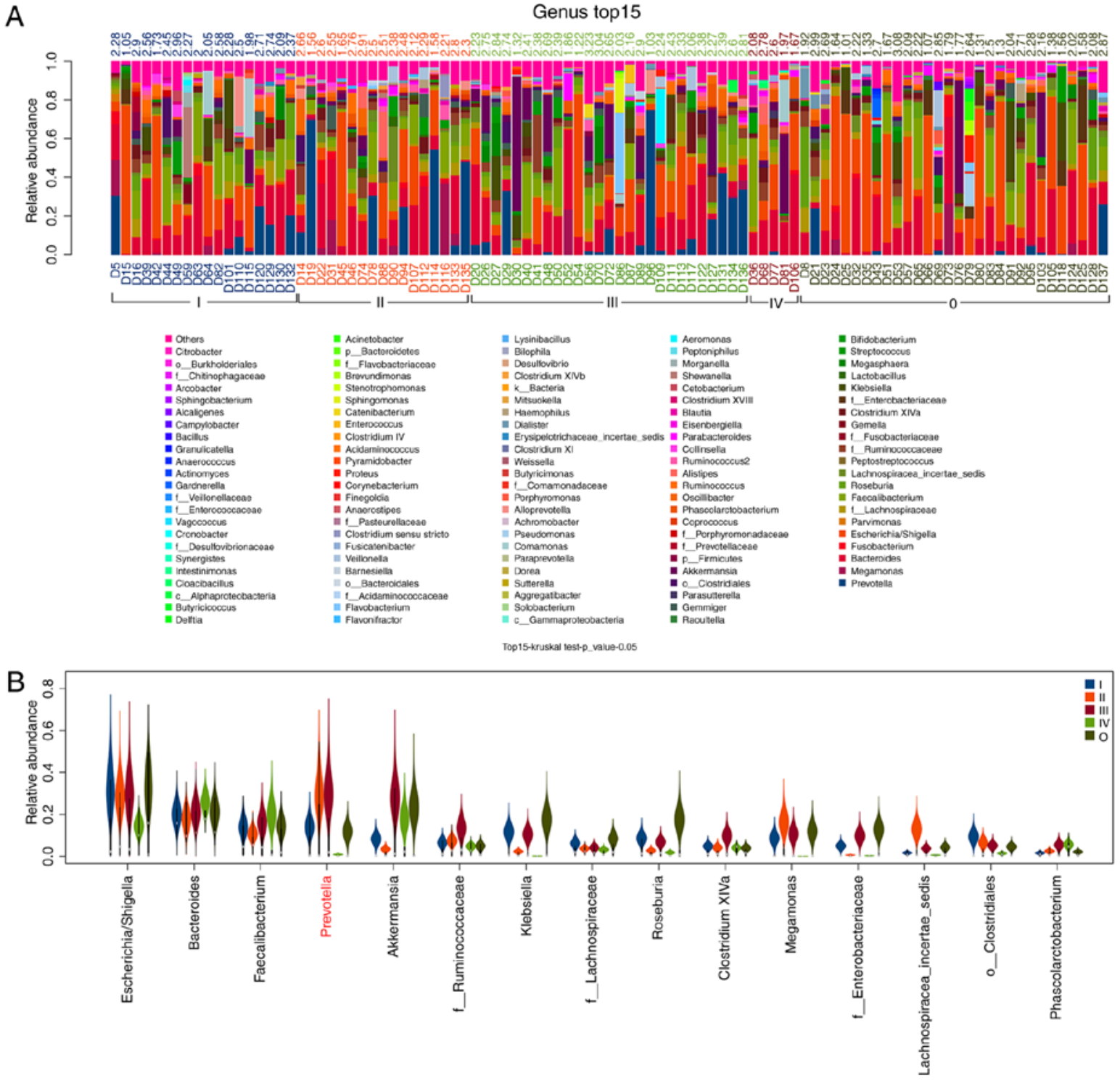

Figure 2. Composition of the fecal microbiota of healthy controls and patients with CRC stages I, II, III and IV at the genus level. (A) The relative abundance of microorganisms comprising the fecal microbiota was determined. Each column represents one fecal sample and the ordinate indicates the relative abundance of taxa. Patients are presented according to disease status, including stages I-IV of CRC and O for healthy controls. (B) The relative abundance of Prevotella within the fecal gut microbiota differed between healthy controls and patients with CRC of stages I-IV. CRC, colorectal cancer.

of PC1 and PC2, accounting for 12.7 and $9.5 \%$ of the total variations, respectively (Fig. 3A). PCoA according to weighted UniFrac metrics confirmed the aforementioned results, indicating differences between patients with CRC of stages I-IV and healthy controls; however, the results of stage I-IV CRC patients overlapped with healthy controls and could not be separated well from the PC1 and PC2 values (9.99 and $8.05 \%$ of the interpreted variance, respectively; Fig. 3B).

Gene functional classifications using the KEGG database. Based on KEGG database analysis, gene functional classifications of the top 50 assembled unigenes are presented in Fig. 4. Significant differences in the function of the unigenes were reported between healthy controls and patients with CRC of stages I-IV $(\mathrm{P}<0.05)$, including 'transporters', 'ribosome', 'transcription factors', 'ABC transporters', 'peptidases', 'translation proteins', 'translation factors', 'signal transduction mechanisms', 'peptidoglycan biosynthesis' and 'glycine, serine and threonine metabolism'.
The bacterial communities in fecal samples of patients with CRC and healthy controls were investigated; all fecal samples comprised 28 phyla, 61 classes, 99 orders, 191 families and 442 genera. The bacterial phyla of all gut microbiota were analyzed. The differences at the phylum level in the fecal gut microbiota were determined between healthy controls and patients with CRC of stages I-IV. The results revealed that, compared with the healthy controls (stage $\mathrm{O}$ ), the relative abundance of Fusobacteria $(7.351 \pm 21.960$ vs. $16.251 \pm 24.25$ $3 \%$; $\mathrm{P}=0.028$ ) was increased in patients with stage I CRC. In addition, the relative abundance of Lentisphaerae was greater in patients with stage II CRC than those of stage I $(0.169 \pm 0.504$ vs. $0.370 \pm 1.155 \% ; \mathrm{P}=0.031)$. Compared with stage II CRC patients, the abundance of Fusobacteria $(16.731 \pm 31.397$ vs. $7.103 \pm 15.111 \% ; \mathrm{P}=0.043)$ was significantly decreased in patients with stage III CRC (Fig. S2); that of the phylum Verrucomicrobia $(10.141 \pm 19.264$ vs. $90.380 \pm 158.505 \%$; $\mathrm{P}=0.035$ ) was significantly increased in patients with stage III 
A

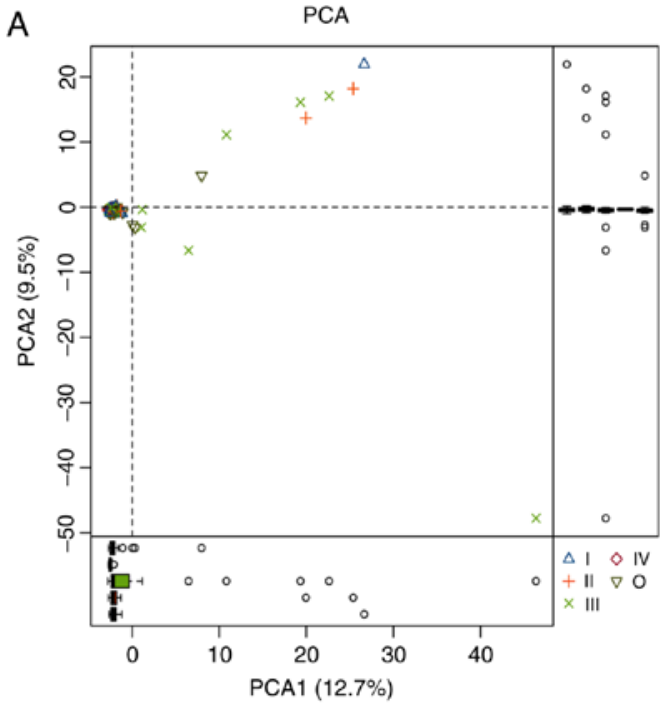

B

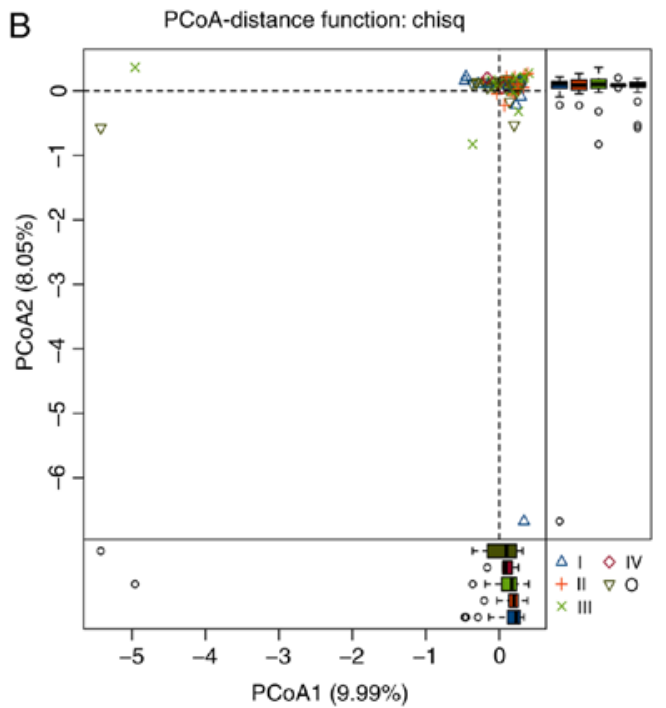

Figure 3. Visualization of the PCA and PCoA results for the fecal microbial samples from patients with CRC stages I-IV and healthy controls. (A) PCA scores map based on the relative abundance of optional taxonomic units (97\% similarity level). (B) PCoA diagram based on weighted UniFrac metrics. PCA, principal component analysis; PCoA, principal coordinate analysis; O, healthy controls; I, stage I colorectal cancer, II, stage II colorectal cancer; III, stage III colorectal cancer; IV, stage IV colorectal cancer.

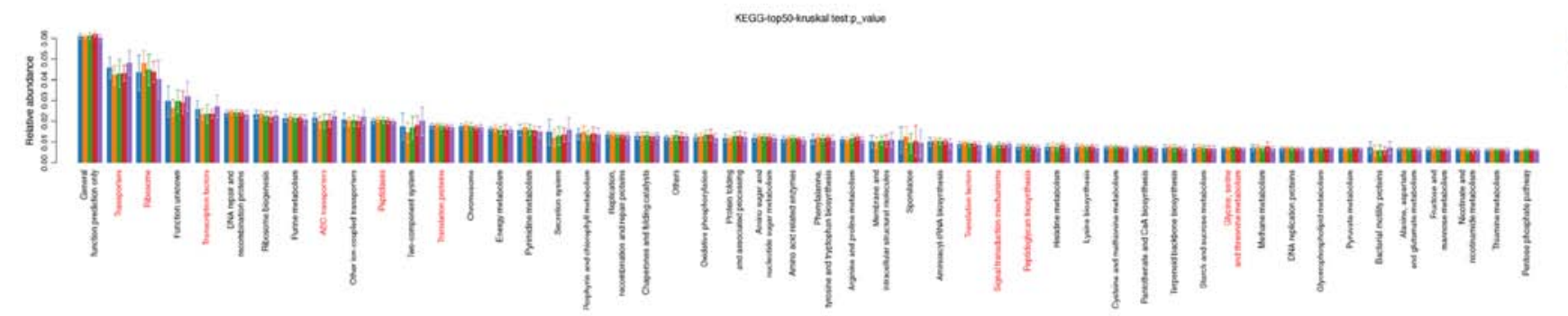

Figure 4. Gene functional classifications of the top 50 assembled unigenes by KEGG enrichment analysis. Significantly different functional gene categories among healthy controls and patients with CRC stages I-IV are highlighted in red. P $<0.05$. O, healthy controls; I, stage I colorectal cancer; II, stage II colorectal cancer; III, stage III colorectal cancer; IV, stage IV colorectal cancer; KEGG, Kyoto Encyclopedia of Genes and Genomes.

CRC (Fig. S2). On the contrary, no differences between patients with CRC of stages III and IV were reported.

At the genus level, the bacterial genera of the gut microbiota were analyzed. The results indicated significant differences in the microbial composition of dominant genera between CRC patients and healthy controls (Fig. 5). Compared with healthy controls, the genera Prevotella $(0.021 \pm 0.063$ vs. $0.095 \pm 0.172$; $\mathrm{P}=0.025)$, Collinsella $(1.671 \pm 2.595$ vs. $6.002 \pm 10.466 \%$; $\mathrm{P}=0.028)$, Odoribacter $(0.436 \pm 0.894$ vs. $1.173 \pm 1.866 \%$; $\mathrm{P}=0.043)$, Peptostreptococcus $(0.070 \pm 0.149$ vs. $2.262 \pm 5.794 \%$; $\mathrm{P}=0.042)$ and Lachnoanaerobaculum $(0.003 \pm 0.012$ vs. $0.027 \pm 0.055 \% ; \mathrm{P}=0.021)$ were significantly enriched in patients with CRC. However, the abundances of Escherichia-Shigella $(0.262 \pm 0.273$ vs. $0.126 \pm 0.183 ; \mathrm{P}=0.005)$, Cronobacter $(0.101 \pm 0.320$ vs. $0.014 \pm 0.066 \% ; \mathrm{P}=0.035)$ and Sporobacter $(0.003 \pm 0.012$ vs. $0 \%$; $\mathrm{P}=0.033)$ were significantly reduced in patients with CRC.

In addition, the effects of different tumor stages on the composition of the gut microbiota were investigated in healthy controls and patients with CRC of stages I-IV. The results revealed that, compared with the healthy controls (stage $\mathrm{O}$ ), the relative abundances of Ruminococcus $(8.803 \pm 14.593$ vs. $23.469 \pm 31.995 \%$; $\mathrm{P}=0.035)$,
Collinsella $(1.671 \pm 2.595$ vs. $9.496 \pm 17.125 \% ; \mathrm{P}=0.017)$ Parvimonas $(0.047 \pm 0.149$ vs. $1.737 \pm 3.013 \% ; \mathrm{P}=0.004)$ and Peptostreptococcus $(0.070 \pm 0.012$ vs. $23.469 \pm 31.995 \%$; $\mathrm{P}=0.017)$ were significantly enriched in stage I CRC patients (Fig. 6). Furthermore, Paraeggerthella (0 vs. $0.100 \pm 0.265 \%$; $\mathrm{P}=0.044$ ) was only detected in patients with stage I CRC. The relative abundance of Hydrogenoanaerobacterium was determined and detected in patients with stage II CRC, but not stage I patients ( 0 vs. $0.012 \pm 0.022 \% ; \mathrm{P}=0.030)$. On the contrary, compared with patients with stage II CRC, the abundance of Akkermansia was significantly increased in those with stage III CRC (Fig. 6). Nevertheless, compared with patients with stage III CRC, the genera Phascolarctobacterium, Parasutterella, Comamonas, Cloacibacillus and Olsenella were relatively enriched in those with stage IV CRC; the abundances of Alistipes, Blautia, Eisenbergiella, Intestinimonas, Eggerthella and Anaeroglobus were significantly decreased in patients with stage IV CRC (Table II). Furthermore, Methylophilus $(0.003 \pm 0.012$ vs. $0 \% ; \mathrm{P}=0.017)$ and Synergistes $(0.003 \pm 0.012$ vs. $0 \% ; \mathrm{P}=0.017$ ) were detected only in stage III CRC patients (data not shown).

Additionally, the key microorganisms of the gut microbiota in the proximal (right) and distal (left) segments of 

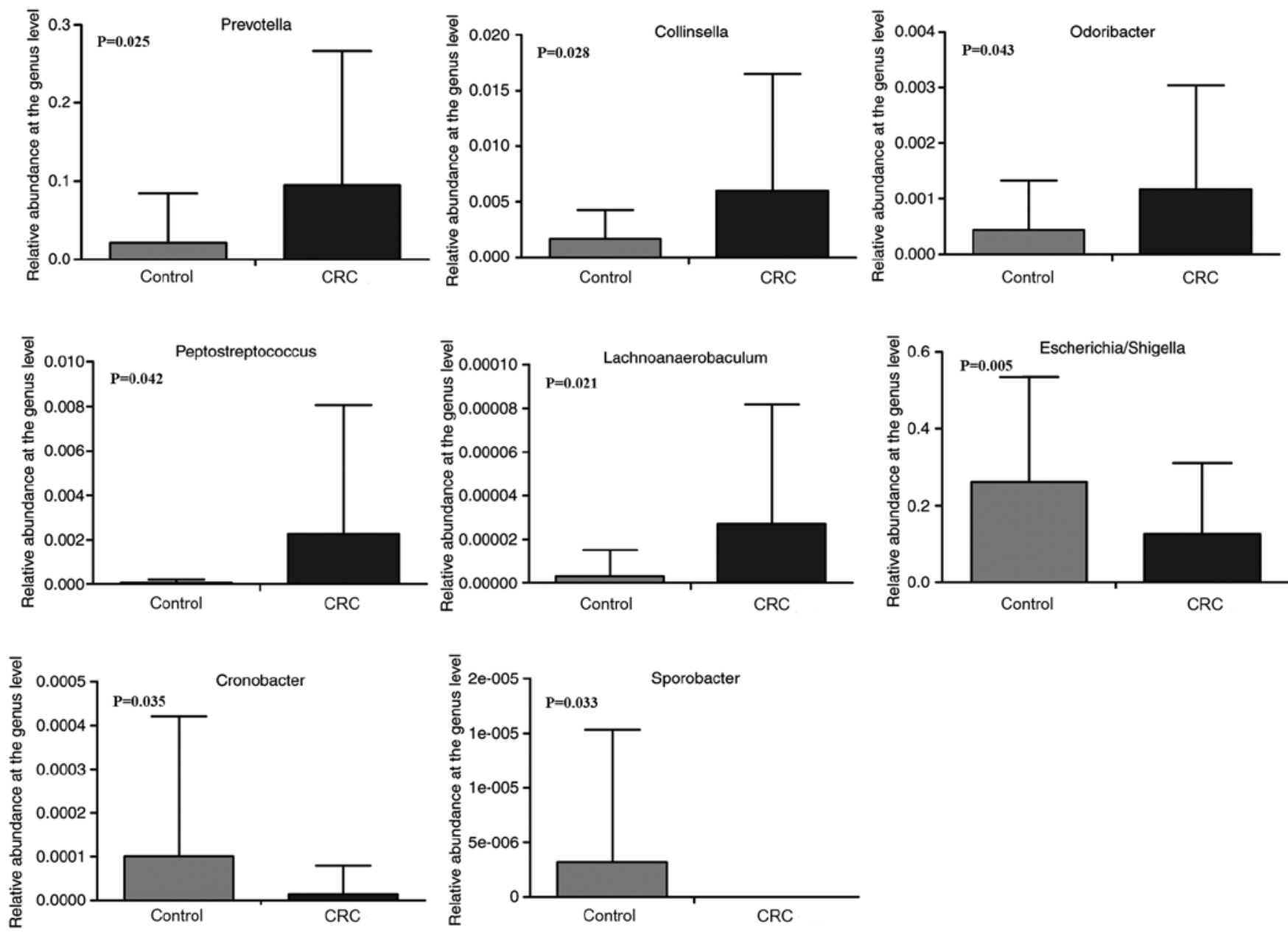

Figure 5. Relative abundance of genera in fecal samples of patients with CRC and healthy controls. CRC, colorectal cancer.

patients with CRC were analyzed. At the genus level, the abundance of 442 genera in the distal (left) and proximal (right) segments were inversely associated with splenic flexure syndrome. The present study reported that these genera Veillonella, Granulicatella and Coprobacter were more abundant in the proximal (right) segment than in the distal (left) segment and the differences were statistically significant $(\mathrm{P}<0.05$; Table III). By contrast, the genera Sneathia $(0$ vs. $0.024 \pm 0.065 \% ; \mathrm{P}=0.014)$, Acetanaerobacterium (0 vs. $0.005 \pm 0.014 \% ; \mathrm{P}=0.036$ ), Phocaeicola (0 vs. $0.007 \pm 0.023 \%$; $\mathrm{P}=0.048)$ and Anaerofustis (0 vs. $0.007 \pm 0.023 \% ; \mathrm{P}=0.047$ ) were detected only in the proximal (right) segments (data not shown).

\section{Discussion}

$\mathrm{CRC}$ is a major public health problem worldwide; the cause of CRC is largely due to a combination of genetic susceptibility and lifestyle and environmental factors (21). In recent years, differences in the composition of the gut microbiota were reported to potentially affect the initiation and progression of $\mathrm{CRC}$; the dysbiosis of the symbiotic microbiota may also be associated with systemic inflammatory disorders and various types of cancer $(22,23)$. At present, tumor staging is the most important prognostic indicator for patients with CRC and various strategies have been developed for the TNM staging of certain types of cancer (24). In addition, the 16S rRNA gene sequencing method has been widely used as an effective tool to analyze the global microbial community (25). In the present study, the 16S rRNA sequencing approach was applied to examine differences in the gut microbiota between patients with CRC of stages I-IV and healthy controls, and the proximal (right) and distal (left) segments relative to the splenic flexor muscle.

The richness and diversity of the gut microbiota of patients with CRC and healthy controls was compared. The richness was represented by the Chaol index and diversity was expressed by the Shannon and Simpson indexes. No significant differences in the Chao1, Shannon and Simpson indexes were observed between CRC patients of stages I-IV and healthy controls. The possible cause for these findings may be that the microbiota of fecal samples in CRC patients of stages I-IV and healthy controls were examined at relatively close stages, and the sample sizes could be insufficient or unequal. The results also demonstrated no significant differences in the Chaol index between patients with CRC and healthy controls but were reported for the Shannon and Simpson indexes. This indicated the increased diversity of the gut microbiota of patients with CRC compared with the healthy controls.

Furthermore, the present study demonstrated that, compared with healthy controls, there were differences in the composition of the gut microbiota of CRC patients, regardless 

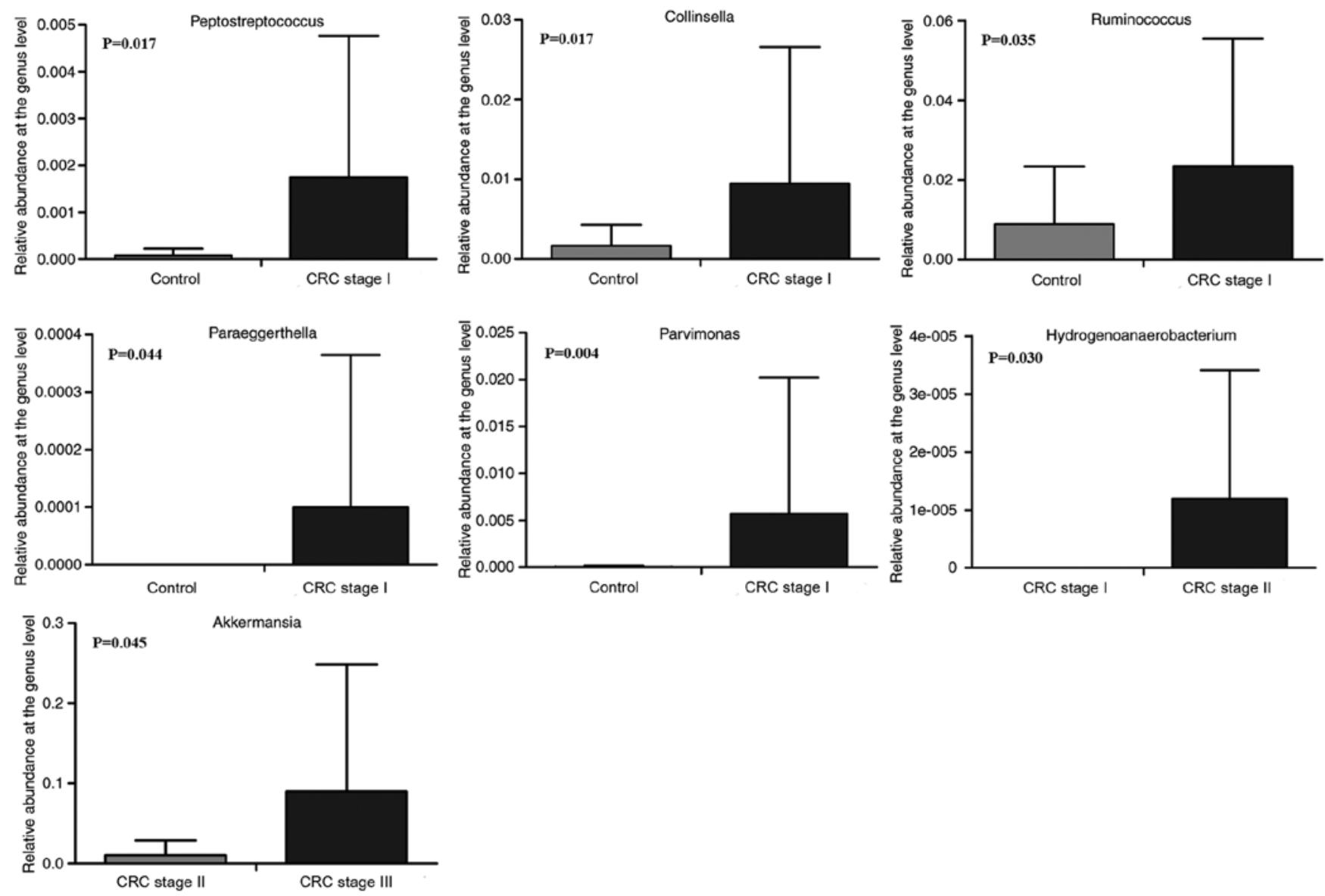

Figure 6. Relative abundance of genera in fecal samples of healthy controls and patients with CRC of stages I-III. CRC, colorectal cancer.

of disease stage. The relative abundance of the top 5 dominant genera Escherichia-Shigella, Bacteroides, Faecalibacterium, Prevotella and Akkermansia differed. At the genus level, compared with healthy controls, the relative abundances of Prevotella, Collinsella and Peptostreptococcus were increased in CRC patients. Previous studies have reported that Prevotella, Peptostreptococcus and other opportunistic bacteria are relatively abundant at tumor sites $(17,26)$, which was consistent with the gut microbiota of fecal samples analyzed in the present study. The genus Peptostreptococcus, formerly known as Peptococcus, has recently been determined to be involved in CRC and was identified to promote the proliferation of CRC cells by inducing the biosynthesis of intracellular cholesterol $(27,28)$. Additionally, patients with symptomatic atherosclerosis, classified as atherosclerotic plaques associated with carotid stenosis that lead to cerebrovascular events, exhibited a high abundance of Collinsella (29). The present study identified that Escherichia-Shigella was significantly reduced in CRC patients. Of note, the number of Escherichia-Shigella in the tumor tissue of patients with CRC was determined to be reduced (11); however, the increased abundance of Escherichia-Shigella suggested its association with the pathogenesis of inflammatory bowel disease (IBD) (30). These results indicate that Escherichia-Shigella leads to different manifestations in patients with CRC and IBD, yet further investigation is required to determine whether these strains are pathogenic in CRC. In addition, CRC-associated Prevotella has also been reported to be linked with variations in mucosal gene-expression profiles, which could be used as a tool for screening CRC in high-risk populations $(5,31)$. These findings and the results of the present study suggested that Prevotella, Collinsella, Peptostreptococcus and Escherichia-Shigella may contribute to variations in the gut microbiota of patients with CRC. In addition, gene and species markers may indicate alterations in the microbiota during early stages of neoplastic growth, which suggests the potential of sensitive microbial markers of advanced adenomas, such as CRC (16). Nevertheless, the present study reported that the relative abundance of these bacteria was associated with CRC patients; however, the direct link between bacterial imbalance and CRC requires further investigation.

At the genus level, differences were also identified in the gut microbiota among patients with CRC stages I-IV and healthy controls. The results revealed that the relative abundances of Collinsella and Peptostreptococcus were substantially increased in patients with stage I CRC compared with healthy controls (stage $\mathrm{O}$ ), which was consistent with differences in the gut microbiota between healthy controls and all CRC patients. The relative abundance of Ruminococcus was also increased in stage I CRC patients. A previous study demonstrated that, compared with healthy controls and advanced adenomas, Alistipes were enriched in patients with CRC (32). In addition, the abundance of OTU-related Alistipes fnegoldii gradually increased during the development of CRC, P53, K-RAS and $B R A F$, demonstrating that biological evolutionary transformation of gut microbiota, characterized by the increase in DNA 
Table II. Relative abundance of genera in fecal samples and statistical differences in the gut microbiota at the genus level between patients with stage III and IV CRC.

\begin{tabular}{lccc}
\hline Genus & CRC stage III, \% & CRC stage IV, \% & P-value \\
\hline Phascolarctobacterium & $13.563 \pm 14.576$ & $13.756 \pm 24.568$ & 0.033 \\
Parasutterella & $0.785 \pm 1.789$ & $1.378 \pm 2.233$ & 0.010 \\
Comamonas & $0.781 \pm 2.250$ & $1.870 \pm 8.948$ & 0.026 \\
Cloacibacillus & $0.090 \pm 0.257$ & $0.280 \pm 1.069$ & 0.025 \\
Olsenella & $0.003 \pm 0.012$ & $0.042 \pm 0.127$ & 0.021 \\
Alistipes & $24.969 \pm 60.888$ & $8.852 \pm 13.432$ & 0.016 \\
Blautia & $7.467 \pm 8.095$ & $5.138 \pm 4.640$ & 0.047 \\
Eisenbergiella & $2.267 \pm 4.444$ & $0.809 \pm 1.387$ & 0.036 \\
Intestinimonas & $0.305 \pm 1.134$ & $0.239 \pm 0.613$ & 0.027 \\
Eggerthella & $0.234 \pm 0.523$ & $0.153 \pm 0.255$ & 0.026 \\
Anaeroglobus & $0.070 \pm 0.243$ & $0.002 \pm 0.009$ & 0.009
\end{tabular}

Data are presented as the mean \pm standard deviation. $\mathrm{CRC}$, colorectal cancer.

Table III. Relative abundance of genera in the fecal samples and statistical differences in the gut microbiota at the genus level between the distal (left) and proximal (right) segments of patients with CRC.

\begin{tabular}{lccc}
\hline Genus & Distal (left) segment, $\%$ & Proximal (right) segment, $\%$ & P-value \\
\hline Veillonella & $1.548 \pm 3.792$ & $16.747 \pm 33.805$ & 0.004 \\
Granulicatella & $0.191 \pm 0.300$ & $0.468 \pm 0.630$ & 0.018 \\
Coprobacter & $0.058 \pm 0.161$ & $0.716 \pm 2.135$ & 0.042 \\
\hline
\end{tabular}

Data are presented as the mean \pm standard deviation. CRC, colorectal cancer.

damage-causing bacteria, is associated with tumorigenesis in CRC models (33). On the contrary, the present study identified that, compared with stage III CRC, the relative abundance of Alistipes was decreased in patients with stage IV CRC. This was inconsistent with previous results and could be due to the small sample size of the present study. Therefore, studies involving more samples should be conducted to determine potential differential characteristics of the gut microbiota at different stages of CRC.

Differences in the gut microbiota of the proximal (right) and distal(left)colon segments of patients with CRC were determined. At the genus level, compared with the distal (left) segment, the genera Veillonella, Granulicatella and Coprobacter were highly enriched in the proximal (right) segment. Veillonella dispar was associated with patients with adenocarcinoma (34); however, Sneathia, Acetanaerobacterium, Phocaeicola and Anaerofustis were detected only in the proximal (right) segments, suggesting that these gut microbiota could be related to these regions within patients with CRC.

The present study demonstrated differences in the gut microbiota of stool samples at the genus level between stage I-IV CRC patients and healthy controls, and the proximal (right) and distal (left) segments relative to the splenic flexor muscle. Of note, the sample size of patients within the different groups employed in the present study was relatively small, which may pose certain limitations. For instance, other intestinal bacterial species may not be identified, such as Fusobacterium nucleatum $(F n)$, which is a gram-negative anaerobe that is enriched in the oral cavity, but is rarely detected in other organs under physiological conditions (35). Fn serves an important role in the organization of biofilms in the oral cavity and may be a dominant microbe that creates physical and metabolic scaffolds that support the microbial shift of numerous microbes in developing tumors over time (36). However, this bacterium is not a predominant species in stool samples and has been detected in cancer biopsies of patients with CRC by two independent research groups using whole-genome sequencing techniques (37). Regarding $F n$, its abundance was lower in the present study; bacteria of higher abundance at the genus level were selected for further analysis. Fn has been recently identified as a pathogenic bacteria in CRC (38). Additionally, the present study reported that the levels of $F n$ were increased in tumor tissues compared with in adjacent normal tissues of the same patients, supporting the authors' previous study (39), which revealed its role in promoting the initiation and development of CRC. Future investigations with a larger number of patients are required to determine the effects of $F n$.

In conclusion, despite the low number of samples included in the present study, dysbiosis and increased diversity of the gut microbiota were reported in CRC. In addition, Prevotella, Collinsella, Peptostreptococcus and Escherichia-Shigella were proposed as potential fecal 
markers for the early detection of CRC. The results of the present study also suggest that bacterial communities and CRC might be further investigated for their possible correlations in order to assess the opportunity of detecting colon cancer through the analysis of specific fecal bacterial markers (40). In addition, differences were also reported in the bacterial populations present in the gut microbiota of different sites and at various stages of disease. Therefore, the constituents of the gut microbiota may be associated with the biological characteristics of CRC; however, whether variations in biological disorders are related to the pathogenesis of $\mathrm{CRC}$, or simply the results of competitive bacteria utilization in the tumor microenvironment, remains to be confirmed in larger clinical and experimental studies. Of note, to improve understanding of the occurrence and development of CRC, other candidate pathogens should be investigated in the future using tumor samples of patients with CRC, oral microbiota and related animal models. Furthermore, additional metagenomic data can enable the in-depth analysis of cancer-associated differences in gene function, genomic content and variation. This could serve as a basis for determining the potential mechanisms underlying the roles of the microbiota in the development and progression of cancer $(16,41)$. Future investigations may aid the monitoring of the microbiota in the early detection of CRC.

\section{Acknowledgements}

Not applicable.

\section{Funding}

The present study was funded by the Natural Science Foundation of Zhejiang Province (grant no. LY18H160018) and the Medical and Health Science and Technology Project of Zhejiang Province (grant no. 2017KY360).

\section{Availability of data and materials}

The data used to support the finding of this study are available from the corresponding author upon request.

\section{Authors' contributions}

QS, HD and JL analyzed and interpreted the patient data regarding the patients with colorectal cancer; QS wrote the manuscript; HD, XFC and XBC participated in the experimental study and data analysis; YT, LP and QW participated data collecting and statistical analysis; JL conceived the idea and designed the study as the corresponding authors of this paper; All authors read and approved the final manuscript.

\section{Ethics approval and consent to participate}

The present study was approved by the Research Ethics Committee of The First Affiliated Hospital, College of Medicine, Zhejiang University (approval no. 2016-436). All patients indicated that they had obtained written informed consent in the present study.

\section{Patient consent for publication}

Not applicable.

\section{Competing interests}

The authors declare that they have no competing interests.

\section{References}

1. Siegel RL, Miller KD and Jemal A: Cancer statistics, 2015. CA Cancer J Clin 65: 5-29, 2015.

2. Chen W, Zheng R, Baade PD, Zhang S, Zeng H, Bray F, Jemal A, Yu XQ and He J: Cancer statistics in China, 2015. CA Cancer J Clin 66: 115-132, 2016.

3. Torre LA, Bray F, Siegel RL, Ferlay J, Lortet-Tieulent J and Jemal A: Global cancer statistics, 2012. CA Cancer J Clin 65: 87-108, 2015

4. Russo E, Taddei A, Ringressi MN, Ricci F and Amedei A: The interplay between the microbiome and the adaptive immune response in cancer development. Therap Adv Gastroenterol 9: 594-605, 2016.

5. Flemer B, Lynch DB, Brown JM, Jeffery IB, Ryan FJ, Claesson MJ, O'Riordain M, Shanahan F and O'Toole PW: Tumour-associated and non-tumour-associated microbiota in colorectal cancer. Gut 66: 633-643, 2017.

6. Nakatsu G, Li X, Zhou H, Sheng J, Wong SH, Wu WK, Ng SC, Tsoi H, Dong Y, Zhang N, et al: Gut mucosal microbiome across stages of colorectal carcinogenesis. Nat Commun 6: 8727-8736, 2015.

7. Bufill JA: Colorectal cancer: Evidence for distinct genetic categories based on proximal or distal tumor location. Ann Intern Med 113: 779-788, 1990.

8. Tamas K, Walenkamp AM, de Vries EG, van Vugt MA, Beets-Tan RG, van Etten B, de Groot DJ and Hospers GA: Rectal and colon cancer: Not just a different anatomic site. Cancer Treat Rev 41: 671-679, 2015.

9. Missiaglia E, Jacobs B, D'Ario G, Di Narzo AF, Soneson C, Budinska E, Popovici V, Vecchione L, Gerster S, Yan P, et al: Distal and proximal colon cancers differ in terms of molecular, pathological, and clinical features. Ann Oncol 25: 1995-2001, 2014

10. Shan L, Konstantinov SR, Smits R and Peppelenbosch MP: Bacterial biofilms in colorectal cancer initiation and progression. Trends Mol Med 23: 18-30, 2017.

11. Gao Z, Guo B, Gao R, Zhu Q and Qin H: Microbiota disbiosis is associated with colorectal cancer. Front Microbiol 6: 20, 2015.

12. Wang T, Cai G, Qiu Y, Fei N, Zhang M, Pang X, Jia W, Cai S and Zhao L: Structural segregation of gut microbiota between colorectal cancer patients and healthy volunteers. ISME J 6: 320-329, 2012.

13. Flemer B, Herlihy M, O'Riordain M, Shanahan F and O'Toole PW: Tumour-associated and non-tumour-associated microbiota: Addendum. Gut Microbes 8: 1-5, 2018.

14. Sabino J, Vieira-Silva S, Machiels K, Joossens M, Falony G, Ballet V, Ferrante M, Van Assche G, Van der Merwe S, Vermeire S and Raes J: Primary sclerosing cholangitis is characterised by intestinal dysbiosis independent from IBD. Gut 65: 1681-1689, 2016.

15. Wu N, Yang X, Zhang R, Li J, Xiao X, Hu Y, Chen Y, Yang F, Lu N, Wang Z, et al: Dysbiosis signature of fecal microbiota in colorectal cancer patients. Microb Ecol 66: 462-470, 2013.

16. Russo E, Bacci G, Chiellini C, Fagorzi C, Niccolai E, Taddei A, Ricci F, Ringressi MN, Borrelli R, Melli F, et al: Preliminary comparison of oral and intestinal human microbiota in patients with colorectal cancer: A pilot study. Front Microbiol 8: 2699-2712, 2018.

17. Gao R, Kong C, Huang L, Li H, Qu X, Liu Z, Lan P, Wang J and Qin H: Mucosa-associated microbiota signature in colorectal cancer. Eur J Clin Microbiol Infect Dis 36: 2073-2083, 2017.

18. Hamady M, Walker JJ, Harris JK, Gold NJ and Knight R: Error-correcting barcoded primers allow hundreds of samples to be pyrosequenced in multiplex. Nat Methods 5: 235-237, 2008.

19. Edgar RC: UPARSE: Highly accurate OTU sequences from microbial amplicon reads. Nat Methods 10: 996-998, 2013. 
20. Lozupone C, Lladser ME, Dan K, Stombaugh J and Knight R UniFrac: An effective distance metric for microbial community comparison. Isme J 5: 169-172, 2011.

21. Rodriguez-Castaño GP, Caro-Quintero A, Reyes A and Lizcano F: Advances in gut microbiome research, opening new strategies to cope with a western lifestyle. Front Genet 7: 224, 2017.

22. García-Castillo V, Sanhueza E, Mcnerney E, Onate SA and García A: Microbiota dysbiosis: A new piece in the understanding of carcinogenesis puzzle. J Med Microbiol 65: 1347-1362, 2016.

23. Rajagopala SV, Vashee S, Oldfield LM, Suzuki Y, Venter JC, Telenti A and Nelson KE: The human microbiome and cancer. Cancer Prev Res (Phila) 10: 226-234, 2017.

24. Wei Z, Cao S, Liu S, Yao Z, Sun T, Li Y, Li J, Zhang D and Zhou Y: Could gut microbiota serve as prognostic biomarker associated with colorectal cancer patients' survival? A pilot study on relevant mechanism. Oncotarget 7: 46158-46172, 2016.

25. Caporaso JG, Lauber CL, Walters WA, Berg-Lyons D Lozupone CA, Turnbaugh PJ, Fierer N and Knight R: Global patterns of 16S rRNA diversity at a depth of millions of sequences per sample. Proc Natl Acad Sci USA 108: 4516-4522, 2011.

26. Chen W, Liu F, Ling Z, Tong X and Xiang C: Human intestinal lumen and mucosa-associated microbiota in patients with colorectal cancer. PLoS One 7: e39743, 2012.

27. Yu J, Feng Q, Wong SH, Zhang D, Liang QY, Qin Y, Tang L, Zhao H, Stenvang J, Li Y, et al: Metagenomic analysis of faecal microbiome as a tool towards targeted non-invasive biomarkers for colorectal cancer. Gut 66: 70-78, 2017.

28. Tsoi H, Chu ESH, Zhang X, Sheng J, Nakatsu G, Ng SC, Chan AWH, Chan FKL, Sung JJY and Yu J: Peptostreptococcus anaerobius induces intracellular cholesterol biosynthesis in colon cells to induce proliferation and causes dysplasia in mice. Gastroenterology 152: 1419-1433, 2017.

29. Karlsson FH, Fåk F, Nookaew I, Tremaroli V, Fagerberg B, Petranovic D, Bäckhed F and Nielsen J: Symptomatic atherosclerosis is associated with an altered gut metagenome. Nat Commun 3: 1245, 2012

30. Chen L, Wang W, Zhou R, Ng SC, Li J, Huang M, Zhou F, Wang X, Shen B, A Kamm M, et al: Characteristics of fecal and mucosa-associated microbiota in Chinese patients with inflammatory bowel disease. Medicine (Baltimore) 93: e51, 2014.

31. Purcell RV, Visnovska M, Biggs PJ, Schmeier S and Frizelle FA: Distinct gut microbiome patterns associate with consensus molecular subtypes of colorectal cancer. Sci Rep 7: 11590, 2017.
32. Feng Q, Liang S, Jia H, Stadlmayr A, Tang L, Lan Z, Zhang D, $\mathrm{Xia} \mathrm{H}$, Xu X, Jie Z, et al: Gut microbiome development along the colorectal adenoma-carcinoma sequence. Nat Commun 6: 6528, 2015.

33. Sun T, Liu S, Zhou Y, Yao Z, Zhang D, Cao S, Wei Z, Tan B, Li Y, Lian $Z$ and Wang S: Evolutionary biologic changes of gut microbiota in an 'adenoma-carcinoma sequence' mouse colorectal cancer model induced by 1,2-Dimethylhydrazine. Oncotarget 8 : 444-457, 2017.

34. Kasai C, Sugimoto K, Moritani I, Tanaka J, Oya Y, Inoue H, Tameda M, Shiraki K, Ito M, Takei Y, et al: Comparison of human gut microbiota in control subjects and patients with colorectal carcinoma in adenoma: Terminal restriction fragment length polymorphism and next-generation sequencing analyses. Oncol Rep 35: 325-333, 2016

35. Han YW: Fusobacterium nucleatum: A commensal-turned pathogen. Curr Opin Microbiol 23: 141-147, 2015.

36. Brennan CA and Garrett WS: Gut microbiota, inflammation, and colorectal cancer. Annu Rev Microbiol 70: 395-411, 2016.

37. Kostic AD, Gevers D, Pedamallu CS, Michaud M, Duke F, Earl AM, Ojesina AI, Jung J, Bass AJ, Tabernero J, et al: Genomic analysis identifies association of Fusobacterium with colorectal carcinoma. Genome Res 22: 292-298, 2012.

38. Yan X, Liu L, Li H, Qin H and Sun Z: Clinical significance of Fusobacterium nucleatum, epithelial-mesenchymal transition, and cancer stem cell markers in stage III/IV colorectal cancer patients. Onco Targets Ther 10: 5031-5046, 2017.

39. Sheng QS, He KG, Li JJ, Zhong ZF, Wang FX, Pan LL and Lin JJ: Comparison of gut microbiome in colorectal cancer in paired tumor and adjacent normal tissues. OncoTargets and Therapy, 2019 (In Press).

40. Schloissnig S, Arumugam M, Sunagawa S, Mitreva M, Tap J, Zhu A, Waller A, Mende DR, Kultima JR, Martin J, et al: Genomic variation landscape of the human gut microbiome. Nature 493: 45-50, 2013.

41. Zeller G, Tap J, Voigt AY, Sunagawa S, Kultima JR, Costea PI, Amiot A, Böhm J, Brunetti F, Habermann N, et al: Potential of fecal microbiota for early-stage detection of colorectal cancer. Mol Syst Biol 10: 766, 2014.

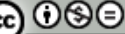

This work is licensed under a Creative Commons Attribution-NonCommercial-NoDerivatives 4.0 International (CC BY-NC-ND 4.0) License. 